\title{
Deployment Techniques of Nodes in WSN and Survey on Their Performance Analysis
}

\author{
Farhad Banoori*, Muhammad Kashif, Muhammad Arslan, Rocky Chakma, Faizan Khan and Abdullah Al Mamun \\ South China University of Technology, China \\ ${ }^{*}$ Corresponding author
}

\begin{abstract}
Wireless Sensor nodes are small, low power consumption nodes which are basically used for sensing, transmitting and receiving the target values to specify nodes. Basically, we deploy nodes to collect information about specific region and to know about current and future results. Wireless Sensor Networks have various applications in many fields e $-\mathrm{g}$ disaster management, biological detection, and traffic, pollution, civil infrastructure monitoring and Surveillance. In this paper, our main purpose is to examines the deployment methodology for a WSN.. This paper also provide general overview and importance on multi-objective strategies, their assumptions, and optimization. In this paper we studies and surveys different sensor deployment methodologies with their ad vantages and disadvantages.
\end{abstract}

Keywords-Network coverage, Network lifetime, Node placement, Node re-positioning, Wireless sensor network.

\section{INTRODUCTION}

This Our main focus is on node deployment in such a best way to achieve our goal i-e to minimize energy consumption and maximize covered region. Here main problem is selectivity of node [1]:

There are many things that we should consider during deployment methodology.

1) No. of sensornodes that are required to fulfill the systemobjectives?

2) how do we most accurately deploy these nodes in such a way to increase systemlife time and performance?

3) How do we manage our the network topology and sensor deployment, when ever some fault occur in a system so that it can remove it easily

Node placement at right place is also big problem as that has been discussed and proved by many researchers as hard complete problem [5-7]. To handle such difficult problem, various method have been offered by scholar and researchers to find its best resolution.Main content of these best possible methodologies is to mainly focus as static, such as to asses fixed network structural topology. Therefore in this paper we consider them as static methods for deploying nodes. Whereas in some situations we need dynamic tuning of nodes position because to achieve the optimal operation of the network but it is also liable on the different network condition and many external factors such as traffic patterns, the load may not be balanced among the nodes causing tailbacks. Due to dynamic behavior of a network energy of existing nodes also reduces.

FIGURE I: DIFFERENT CLASSIFICATIONS OF ST ATIC NODES POSITIONING FOR DEPLOYMENT IN WSN

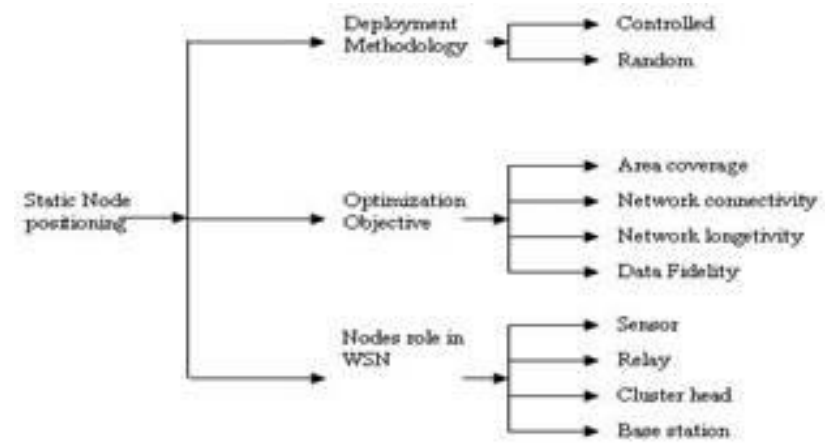

We organized this paper as follows. The next un it described static strategies for node deployment. Different techniques are divided considering the deployment methodology, the primary optimization metrics and the role that the nodes play. Section 3 will give attention to dynamic positioning schemes. At the end we will conclude the survey.

\section{ST ATIC DEPLOYMENT OF NODES}

In WSN, node location have great effect on the performance and its efficiency. Several node location arrangements before network format basically depend on different metrics like coverage area and distance between nodes which are basically not dependent of the network current condition. Generally proper node deployment decrease several complexities e-g data routing, communication. Furthermore in this unit we will focus on some eminent node location approaches and methods from the literature. We categorize them according to function, optimization and methodology.

\section{A. Deployment Methodologies}

We can deploy nodes in our selected region both randomly and deterministically. There are several factors that we should consider during deployment arrangement e-g sensors type, role and external condition in which it plug and play. Deterministic Sensor node placement is achievable and compulsory in such circumstances in which they are exclusive and their location play an important role in their functionality. Due to which WSN life time reduce and 
energy consumption maximize. It is the important reason due to which we deploy different nature of nodes in different cases e-g extremely accurate seismic nodes, submerged WSN applications. Whereas on other side, in some scenarios random distribution of nodes is best to achieve your target values. So in difficult circumstances e-g battle, flooded zone, earth quake etc it is not easy to manage everything according to your requirement. This is particularly true for harsh environments such as a battle field or a disaster region. We can accomplish desired performance targets basically due to node arrangement.

\section{B. Controlled Nodes Deployment}

Although Sensor network assist us in monitoring real world events very closely that's why mostly deterministic model is used for indoor deployment. Examples of Indoor WSN networks examples are Active Sensor Network (ASN) project [6], the Multiple Sensor Indoor Surveillance (MSIS) project at Accenture Technology Labs in Chicago [7] and the Sensor Network Technology projects at Intel [8]. These development lead us in the direction of serving shadowing submissions mostly as safe and protected installations. There are also a lot of problems emerge as cause of polluted water at Sandia National Lab [6,7]. Controlled deployment is appropriate for many purposes such as underwater acoustics and video sensors. In addition to that, some deployment required three-dimensions (3-D) arrangements' which is very proble matic to examine and deploy physically in the field area.

T ABLE I: ASSESSMENT BETWEEN DIFFERENT METHODS AND TECHNIQUE FOR NODESPLACEMENT

\begin{tabular}{|c|c|c|c|c|c|c|c|}
\hline Paper & Application & Space & Deployment & $\begin{array}{l}\text { Node } \\
\text { Type }\end{array}$ & $\begin{array}{l}\text { Primary } \\
\text { Objective }\end{array}$ & $\begin{array}{l}\text { Secondary } \\
\text { Objective }\end{array}$ & Constraints \\
\hline $\begin{array}{l}\text { 1.[5] Alon } \\
\text { Efrat }\end{array}$ & Common & 3-D & Random & Sensor & Coverage & $\begin{array}{l}\text { Min Relay } \\
\text { Count }\end{array}$ & $\begin{array}{l}\text { Fixed Sensor } \\
\text { Count }\end{array}$ \\
\hline $\begin{array}{l}\text { 2.[6] Cheng } \\
\text { Xiuzhen }\end{array}$ & Biomedical & $2-D$ & Deterministic & Relay & $\begin{array}{l}\text { Data } \\
\text { Fidelity }\end{array}$ & $\begin{array}{l}\text { Min Relay } \\
\text { Count }\end{array}$ & Connectivity \\
\hline $\begin{array}{l}\text { 3.[9] Lakshman } \\
\text { Krishnamurthy }\end{array}$ & Common & $2-D$ & Random & $\begin{array}{l}\text { Data } \\
\text { Collector }\end{array}$ & Coverage & $\begin{array}{l}\text { Min Relay } \\
\text { Count }\end{array}$ & Connectivity \\
\hline $\begin{array}{c}\text { 4. [8] Valery A. } \\
\text { Petrushin }\end{array}$ & Observation & $3-D$ & Deterministic & Relay & $\begin{array}{l}\text { Network } \\
\text { Life Time }\end{array}$ & $\begin{array}{l}\text { Min } \\
\text { Sensor } \\
\text { Count }\end{array}$ & $\begin{array}{l}\text { Fixed Sensor } \\
\text { Count }\end{array}$ \\
\hline $\begin{array}{r}5 .[12] \mathrm{CHI}-\mathrm{FU} \\
\text { Huang }\end{array}$ & Outside & $2-D$ & Deterministic & $\begin{array}{l}\text { Data } \\
\text { Collector }\end{array}$ & $\begin{array}{l}\text { Data } \\
\text { Fidelity }\end{array}$ & $\begin{array}{l}\text { Min } \\
\text { Sensor } \\
\text { Count }\end{array}$ & Connectivity \\
\hline $\begin{array}{r}\text { 6.[10] Jian } \\
\text { Tang }\end{array}$ & $\begin{array}{l}\text { Contaminati } \\
\text { on Exposure }\end{array}$ & $2-D$ & Random & Relay & $\begin{array}{l}\text { Network } \\
\text { Life Time }\end{array}$ & $\begin{array}{l}\text { Min } \\
\text { Sensor } \\
\text { Count }\end{array}$ & $\begin{array}{l}\text { Fixed Sensor } \\
\text { Count }\end{array}$ \\
\hline
\end{tabular}

\section{Random Node Distribution}

Controlled arrangement of nodes is sometimes dangerous and difficult to deploy e-g fire burst, earthquake and flooding situations. In these difficult situation then we use randomized deployment to achieve desired goals and targets. Mostly It is extensively predictable that nodes are released through airplane, grenade or clustered ammos. Due to this distribution we accomplished to spread nodes in target zone. Sometime it is difficult to controlled nodes concentration in deployment region. Several exploration developments [7], mostly we consider even sensor dispersal when assessing network performance. We deploy huge number of sensors in our target zone due to its low cost and small size. In 2006 Ishizuka and Aida [11] have studied haphazard sensor dispersal purposes. In 
this section, they try to relate distribution, uniform assignment and R-haphazard assignment procedures. During performing experiments, we noticed that data loss during stalking applications. We observed that R-haphazard placement is a very good approach for error-acceptance metric.

\section{Principal Purposes for Distribution}

The Designers surely design nodes according to need and requirement that's why these nodes should fulfill or achieve desired targets. That's why our main goal is to increase coverage area and communication link between nodes. We also want to include other parameters that can also play an important role in increasing efficiency and reliability[11-13]. It is generally very difficult to achieve your desired goals in randomized deployment. But still there are so many weaknesses or problems exist in deterministic deployment which is not solved in a better way to increase overall system efficiency.

\section{ACTIVE REPLACEMENT OF NODES}

As most of previous designed protocol just consider optimal performance of nodes and most of the research paper have deal with fixed sensor nodes which is basically a big concern for current advance deployment techniques.in addition to that,we consider different parameters and variables when examining performance of sensor nodes in converged area. Different parameter that affect these nodes performance are data rate, no. of hops between specific node and base station. Furthermore, node placement is also very challenging task during dynamic operation in the field because in dynamic situation, some nodes join and leave other nodes in the field so in this operation due to which a lot energy has been wasted. These operations and path length greatly affect network connectivity. For illustration, so when several nodes in the vicinity of the main base-station halt working due to the enervation of their cells, they can use some redundant nodes in their neighborhood zone for network connectivity and continuous operation. Main problem for energetic and active ie freely moving node replacement is described as follows: path finding, data route during node failure and nodes repositioning are important factor which play an important role in dynamic node placement.

\section{RESEARCH ISSUES AND CHALLENGES}

Main purpose of this research paper explains that there are several algorithms proposed to fulfill or achieve random and deterministic deployment. Still many problems are exist that should be consider to increase network connectivity, lifetime and network efficiency. Major issue in random deployment is energy exhaustion whereas in deterministic technique network efficiency is a big challenging issue.

\section{CONCLUSION}

In this paper, we examined and inspected basic major deployment problems, which have close relationship with wireless sensor network. Most importantly we divide deployment of nodes in two major categories i-e random and controlled or deterministic. In controlled methods, node is place in an organized way to achieve network topology and network desired goals. Whereas in random and dynamic deployment, node replacement is a major factor for improving network efficiency. This survey accomplishes that static approaches are further hands-on whereas controlled node deployment are mostly used nodes cost is not a problem. At the end we conclude that deterministic approaches have greater efficiency as compared to random node deployment.

\section{REFERENCES}

[1] L.Buttyan, "Application of wireless sensor networks in critical infrastructure protection: challenges and design options, "IEEE Wireless Communication, Volume No. 17 ,

[2] Issue No. 5, Page No 44 - 49, 2010.

[3] F. P. Kelly, "Rate control for Communication networks: shadow prices, proportional fairness and stability," Journal of Operational Research Society, Volume No. 49, Issue No. 3, Page No. 237-252, March 1998.

[4] S. Ehsan, "Radio and Medium Access Contention Aware Routing for Lifetime Maximization in Multichannel Sensor Networks," IEEE Transactions on Wireless Communications, Volume No.11,Issue No.9, Pages No. 3058-3067 2012.

[5] K. Romer, "The design space of wireless sensor networks," IEEE Wireless Communication, Volume No. 11,Issue No. 6, Pages No. 54-61, 2004.

[6] A. Efrat, "Approximation algorithms for two optimal location problems in sensor networks," in Proceedings of the 2rd International Conference on Broadband Communications, Net works and Systems (Broadnet s"05), Boston, Massachusetts, 7 October 2005.

[7] Cheng, Xiuzhen, "Relay sensor placement in wireless sensor networks," Wireless Networks Volume No.14,Issue No. 3,Pages No. 347-355 June 2008.

[8] S. Poduri, "Sensor network configuration and the curse of dimensionality," in Proceedings of the 3rd IEEE Workshop on Embedded Net worked Sensors(emnets06), 2006.

[9] V.A.Petrushin, "Multiple sensor Indoor Surveillance System," in Proceedings of the 3rd Canadian Conference on Computer and Robot Vision(CRV $\left.{ }^{*} 06\right)$, Que' bec City, Page No.40-40,June 2006.

[10] Lakshman Krishnamurthy, "Design and deployment of industrial sensor net works: Experiences from a semiconductor plant and the North Sea," in Proceedings of the 3rd ACM Conference on Embedded Networked Sensor Systems (Sen-Sys"e5), San Diego, CA,USA Page No.64-75, November 2005.

[11] J. Tang, "Relay node placement in large scale wireless sensor net works," Published in Journal on Computer Communications, Volume No. 29, Issue No.4, Page No.490-501, February 2006.

[12] Mika Ishizuka, "Performance study of node placement in sensor networks," in Proceedings of the 24th International Conference on Distributed Computing Systems Workshops Volume No.7, Hachioji, Tokyo, Japan, April 2004.

[13] CHI-FU Huang, "The Coverage problem in a wireless sensor network," in Proceedings of the workshop on Wireless Sensor Networks and Applications, SanDiego, CA,USA 2003.

[14] Azzedine Boukerche, "A coverage preserving and hole tolerant based scheme for irregular sensing range in wireless sensor networks," in Proceedings of the 49th Annual IEEE Global Communication Conference (Globecom“"06) San Francisco, CA, Page No.1 -5 2006. 